wrist, 2-3 MCP, Knee, tibio-talar and 2-3 MTP joints, and the following tendons: carpal extensor and flexor tendons, tibial posterior and peroneal. For scoring structures, three methods were tested: semiquantitative (0-3 GS +0-3 PD), dichotomous (0/1 GS +0/1 PD), and qualitative ( $0 / 1$ based on algorithm [image 1]). All showed strong correlation with activity measures ( $r$ o $\geq 0.60$ ), and reliability (ICC 0.89 to 0.93). The most feasible index, qualitative, was chosen.

The proposed formula for USAS was: $U S A S=N^{\circ}$ swollen joints + US score+CRP

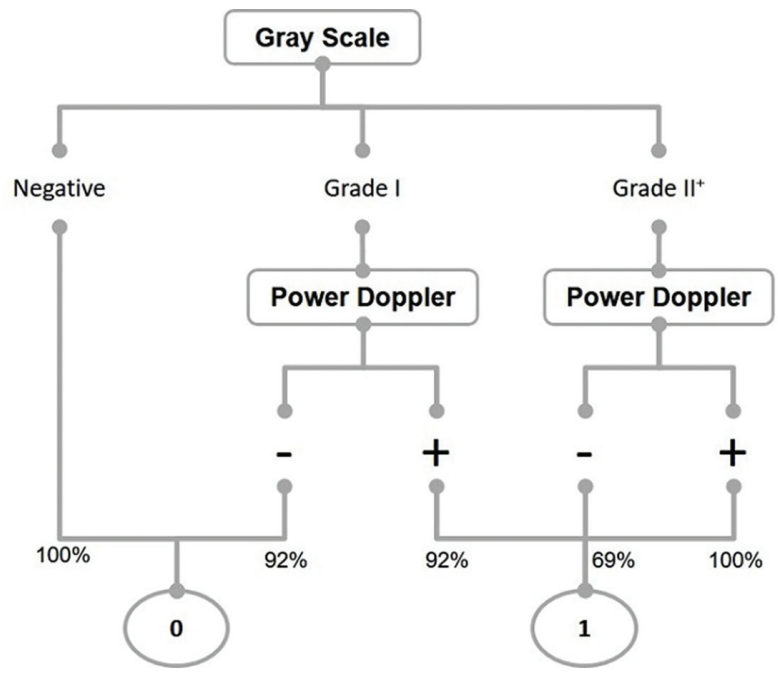

Abstract AB1195 - Figure 1

Conclusions: USAS is a valid and reliable measure of inflammation in RA equal to the sum of 28 swollen joint count, a simplified (0/1) US assessment of 11 structures and CRP.

Disclosure of Interest: None declared

DOI: 10.1136/annrheumdis-2018-eular.3824

\section{AB1196 ROLE OF CAPILLAROSCOPY IN THE STUDY OF THE RAYNAUD PHENOMENON IN CONDITIONS OF CLINICAL PRACTICE}

J. Uceda ${ }^{1}$, R. Hernandez ${ }^{1}$, C. Almeida ${ }^{2}$, J.L. Marenco ${ }^{1} .{ }^{1}$ Rheumatology;

${ }^{2}$ Estadistic, University Valme Hospital, Seville, Spain

Background: The Raynaud phenomenon (RP) is very common throughout the world, especially in cold climates, affecting approximately $3 \%-5 \%$ of the population. Most RPs are primary. The risk of the onset of autoimmune disease associated with RP (ADRP) is between $6 \%$ and $12 \%$, and the diagnosis is usually made within 2 years of its onset. Capillaroscopy is a simple, innocuous and inexpensive technique that is very useful in the study of RP

Objectives: The primary goal was to evaluate the capillaroscopic findings in patients with RF as the only symptom and establish their relationship between the subsequent development of ADRF.

Methods: We conducted an observational, descriptive, cross-sectional study of a random sample of 98 patients with RP as the main manifestation, selected from within the capillaroscopy consult of our Unit. The capillaroscope used was a stereomicroscope (Stereoscope), with a triocular head, zoom range from $1 x$ to $4 x$ magnification, with cold light illuminator and high resolution ocular camcorder. Periungual capillaroscopy was performed in 3rd, 4th and 5thfinger of the right and left hands in each patient and always by the same rheumatologist. We described the sociodemographic variables of patients, and analytical results of the antinuclear antibodies (ANA). After an initial statistical exploration of the data, the same are described. The quantitative variables are expressed as means and standard deviations and if the distributions are asymmetric with medians and quartiles. The qualitative variables with percentages. To assess the validity of capillaroscopy as a diagnostic test, we determined the sensitivity, specificity, and positive and negative predictive value of the test.

Results: $73.5 \%$ were women, with an average age of 45.7 years. The median time of evolution of the RP until the capillaroscopy was 2.5 years. $76 \%$ of the patients were ANA negative.
The evaluation of the predictive capacity of capillaroscopy for the development of EARP yields the following results:

Abstract AB1196 - Table 1

\begin{tabular}{lc}
\hline Sensibility & $83,3 \%{ }^{62,6-95,2}$ \\
\hline Specificity & $78 \%^{66,8-86,9}$ \\
Predictive positive value & $34,1 \%\left(^{24,5-45,3}\right.$ \\
Predictive negative value & $97,1 \%\left(^{93,3-98,8}\right.$ \\
\hline
\end{tabular}

When analysing the relationship between the presence of ANA and the association with ADRF we found that it was independent $(p=0.002)$ of the result of capillaroscopy, being the risk of developing the disease in a patient with positive ANA 8.5 times higher than in an ANA negative patient.

Conclusions: Capillaroscopy in patients with RP has a high predcitive negative value, which allows us to estimated, with high reliability, the association of this phenomenon with autoimmune disease in patients with normal capillaroscopic patterns. Given the results of our study, capillaroscopy should be protocol in the RP study

Disclosure of Interest: None declared

DOI: 10.1136/annrheumdis-2018-eular.5759

\section{AB1197 1 MULTIPARAMETRIC ANALYSIS OF CONNECTIVE TISSUE DISEASE SPECIFIC AUTOANTIBODIES USING A SPOT IMMUNOASSAY (SERASPOT $\left.{ }^{\circledR} A N A\right)$}

K. Conrad ${ }^{1}$, M. Rejzek ${ }^{1}$, M. Aringer ${ }^{2}$, S. Rudolph ${ }^{3}$, L. Unger ${ }^{4}$, K. Lüthke $^{5}$, A. Gräßler ${ }^{6}$, N. Röber ${ }^{1} .{ }^{1}$ Medical Faculty of the TU Dresden, Institute of Immunology; ${ }^{2}$ University Hospital Carl Gustav Carus Dresden, Department of Medicine III, Dresden; ${ }^{3}$ Immune Center Chemnitz, Chemnitz; ${ }^{4}$ Municipal Hospital Dresden-Friedrichstadt, Department of Medicine I; ${ }^{5}$ Medical Practice of Internal Medicine and Rheumatology, Dresden; ${ }^{6}$ Medical Practice Internal Medicine and Rheumatology, Pirna, Germany

Background: Because autoantibody profiling compared to single autoantibody determination is more relevant for diagnostics, differential diagnostics and even prognostics of the different kinds of connective tissue diseases (CTD), cost and time saving multiplex assays are more and more used in routine practice. ${ }^{1}$

Objectives: Evaluation of the diagnostic relevance of the SeraSpot ANA assay (Seramun Diagnostica GmbH, Heidesee, Germany), a novel spot immunoassay for multiplex analysis of the main connective tissue disease (CTD) specific autoantibodies (AABs) against dsDNA, histone, nucleosome, Scl-70, U1-RNP, Sm, PCNA, RibP, Ro52/TRIM21, Ro60, La/SS-B, CENP-B, Jo-1, PM/Scl-100, and $\mathrm{Ku}$ ) based on autoantigens immobilised in microtiter plates.

Methods: AAB profiles using the SeraSpot ANA assay were determined in sera of 381 patients with CTD and 202 apparently healthy individuals (AHI). The CTD patients comprises 105 SLE, 117 systemic sclerosis (SSc), 32 Sjögren's syndrome (SjS), 58 idiopathic inflammatory myopathies (IIM), 5 mixed connective tissue disease (MCTD), and 64 undifferentiated connective tissue disease patients (UCTD).

Results: At least one CTD associated AAB was positive in $88.2 \%$ of the tested CTD patients. A high diagnostic specificity for CTD above $95 \%$ compared to $\mathrm{AHI}$ were found for antibodies to dsDNA, RibP, Sm, Ro60, Ro52, CENP-B, Scl70, PM/ $\mathrm{Scl}-100, \mathrm{Ku}$ and Jo-1. Excluding low-titre reactivity, the specificity of U1-RNP, nucleosome, histone and La/SS-B antibodies was also very high (96.5\%-98\%) regarding CTD diagnosis. The highest specificities vs. AHI were found for antiSm, -Ro60, -RibP and -Jo1 antibodies (99.5\%), followed by anti-CENP-B (99\%), dsDNA (98,5\%), -Ku (98.5\%) and -Ro52 antibodies (98\%). Regarding SLE, 104 (99\%) were positive for SLE-associated AABs. Anti-dsDNA antibodies were most frequently found $(88.6 \%)$. The highest specificities $(98.5 \%-99.5 \%)$ for SLE compared to AHI were found for anti-dsDNA, -RibP, -Sm, and -Ro60 antibodies. SjS relevant AABs against Ro60, Ro52 and La/SS-B were seen in $81.3 \%, 84.4 \%$ and $46.9 \%$ of the SjS patients, respectively. The diagnostic specificity of Ro60 antibodies for SLE and SjS compared to other SARD (excluding UCTD) is $96.8 \%$ and 99.5\%-100\% compared to AHI. SSc associated AAB against Scl-70, CENP-B, $\mathrm{PM} / \mathrm{Scl}-100$ and $-\mathrm{U} 1-\mathrm{RNP}$ were found in $53.0 \%, 20.5 \%, 8.6 \%$, and $13.7 \%$ of the included SSc cases, respectively, with diagnostic specificities between $96 \%$ and 99\%. AABs against Jo-1, PM/Scl-100, U1-RNP, Ro52 and Ku were positive with high specificity $(98.4 \%-99.5 \%$ ) in $29.3 \%, 10.3 \%, 19 \%$ (100\% of MCTD), $27.6 \%$ and $10.3 \%$ of IIM patients, respectively. 
Conclusions: In combination with the HEp-2 cell assay, the SeraSpot ANA assay can be used as a novel cost-effective multiplex assay for the serological confirmation of CTDs.

\section{REFERENCE:}

[1] Mahler M, Meroni PL, Bossuyt X, Fritzler MJ. Current concepts and future directions for the assessment of autoantibodies to cellular antigens referred to as anti-nuclear antibodies. J Immunol Res 2014; Article ID 315179, doi.org/10.1155/2014/315179

Disclosure of Interest: None declared

DOI: 10.1136/annrheumdis-2018-eular.5594

\section{\begin{tabular}{|l|l}
\hline AB1198 & THE DIFFERENCES OF THE DISTRIBUTION OF
\end{tabular} FEEDING VESSELS AND BONE SURFACE IRREGULARITY BETWEEN YOUNG AND ELDERLY ADULTS IN WRIST JOINTS OF HEALTHY VOLUNTEERS BY MUSCULOSKELETAL ULTRASOUND (MSKUS)}

K. Misaki ${ }^{1}, \mathrm{~K} . \mathrm{Ikeda}^{2}$, K. Inoue ${ }^{1}$, Y. Imaizumi ${ }^{3} .{ }^{1}$ Department of Rheumatology, KITA-HARIMA MEDICAL CENTER, Ono; ${ }^{2}$ Department of Allergy and Clinical Immunology, Chiba University, Chiba; ${ }^{3}$ Department of Orthopaedic surgery, KitaHarima Medical Center, ono, Japan

Background: Synovial vascularity as measured by power Doppler (PD) of MSKUS is correlated to rheumatoid arthritis disease activity, and PD signal reveals the prevalence of subclinical synovitis overlooked on physical examination. It is often difficult to distinguish bone erosion from normal concave surface of the bone, and it is necessary for us to be familiar with these normal structures well in evaluating disease activity by using MSKUS. Here we examine the age-specific differences of normal feeding vessels and bone surface irregularity between in wrist joints.

Objectives: To elucidate the differences of distribution of feeding vessels and bone surface irregularity in wrist joints both young and older adults among healthy volunteers.

Methods: The dorsal side of wrist joints was scanned with 2D-probe in healthy volunteers (young $<50$ y.o., elder $\geq 50$ y.o). The distribution of feeding vessels in the capsule and the extensor(E.) tendon sheath(TS), and the evaluation of bone surface irregularity at lunate(Lu) were examined. The comparative review between young and elderly adults was validated.

Results: The distribution of feeding vessels in younger healthy volunteers $(n=30$ : mean age $32.2 \pm 8.0$ y.o.) vs elderly healthy volunteers $(n=21$ :mean age 66.0 \pm 7.2 y.o.) were near-Trapezoid (Rt $100.0 \%$ vs $100.0 \%$, Lt $100.0 \%$ vs $100.0 \%$ : $\mathrm{p}=1.00$ ), E. digitorum TS(Rt86.7\% vs $81.0 \%: \mathrm{p}=0.59, \mathrm{Lt} 66.7 \%$ vs $76.2 \%: \mathrm{p}=0.47$ ), $\mathrm{E}$ digiti minimi TS(Rt30.0\% vs $52.4 \%: \mathrm{p}=0.11$, Lt $30.0 \%$ vs $66.7 \%: \mathrm{p}=0.0089$ ), nearCapitate (Rt23.3\% vs $42.9 \%: p=0.14$, Lt30.0\% vs $47.6 \%: p=0.21$ ), near-TFCC (Rt $16.7 \%$ vs $19.0 \%$ : $\mathrm{p}=0.83$, Lt $30.0 \%$ vs $38.1 \%$ : $\mathrm{p}=0.56$ ), distal radial side of radiocarpal joint(Rt20.0\% vs $42.9 \%$ : $p=0.08$, Lt $23.3 \%$ vs $28.6 \%: p=0.68$ ), distal end of Ulna(Rt10.0\% vs $42.9 \%: \mathrm{p}=0.006$. Lt $16.7 \%$ vs $28.6 \%: \mathrm{p}=0.31$ ). feeding vessels from vascular channels were depicted at $\mathrm{Lu}(\mathrm{R} t 53.3 \%$ vs $52.4 \%: \mathrm{p}=0.95$, Lt 46.7 vs $66.7 \%: \mathrm{p}=0.16$ ), Radius(Rt20.0\% vs $33.3 \%: \mathrm{p}=0.29$, Lt $16.7 \%$ vs $23.8 \%: \mathrm{p}=0.54$ ), Triquetrum (Rt10.0\% vs $42.9 \%$ : $=0.0057$, Lt16.7\% vs $33.3 \%: p=0.17$ ) and Capitate(Rt6.7\% vs $33.3 \%: p=0.013$, Lt10\%vs33.3\%:p=0.0395). The bone surface irregularity as a transverse diameter (Mean \pm S.D.) at Lu of dominant hand in both groups were $1.26 \pm 0.33$ vs $1.14 \pm 0.2 \mathrm{~mm}: \mathrm{p}=0.21$, respectively.

Conclusions: The frequency of feeding vessel's distributions in elderly adults were significantly higher at E.digiti minimi TS, distal end of Ulna and Triquetrum/ Capitate vascular channels compared to those of younger adults. It is suggested that these differences are crucial to evaluate the age-specific synovitis with ultrasound.

Disclosure of Interest: None declared

DOI: 10.1136/annrheumdis-2018-eular.3850

\section{AB1199 DYNAMIC ULTRASOUND FOR MULTILEVEL EVALUATION OF MOTION AND POSTURE IN LOWER EXTREMITY AND SPINE}

R.V. Bubnov ${ }^{1,2}$, L. Kalika ${ }^{3}$, L. Babenko ${ }^{2} .{ }^{1}$ Diagnostic and interventional ultrasound Clinical hospital "Pheophania", ${ }^{2}$ Interferon, Zabolotny Institute of Microbiology and Virology, National Academy of Sciences of Ukraine, Kyiv, Ukraine; ${ }^{3}$ https:// nydnrehab.com/, New York Dynamic Neuromuscular Rehabilitation and Physical Therapy, New York, USA

Background: Evaluation of motion and posture is a crucial task in management patient with rheumatic diseases and pain. Integrative protocol including multilevel assessment of intrinsic/extrinsic muscles postural imbalance for disease staging and treatment efficacy control has not been finally developed.
Objectives: The aim was to study feasibility of multilevel motion and posture analysis using dynamic M-mode ultrasound in foot, ankle, gluteus region, pelvis and spine.

Methods: We included 21 patients (13 females, aged $18-52$ y.o.) with clinically diagnosed reduced motility in spine, pelvis and lower extremity levels due to detected back leg, pain, muscle spasticity, joints effusion, tissue swelling, etc. Another healthy 20 patients (aged 18-50 y.o.) without movement rstriction and pain were controls. We conducted precise physical tests, extensive neuromuscular ultrasound (US) using M-mode to evaluate muscle thickness, CSA and motion in intervetrebral spaces, pelvis, intrinsic/extrinsic muscles in foot and ankle gluteus region and pelvis, central and peripheral trigger points identification.

Results: We obtained sufficient quality panoramic scans on leg using convex 5 $8 \mathrm{MHz}$ probe in 2 approaches to evaluate structure and motion of extrinsic/intrinsic portion of muscles during one session. Thickness measurements of peroneal portion, plantar intrinsic foot muscles on the plantar surface in two transverse positions and one longitudinal using linear probe; contractility using M-mode tested in walking were most representative data. We evaluated different patterns of decreasing motility, contractility (muscle contracted/rested thickness) on M-mode during functional tests and walking in all levels in group $1(p<0.05)$. We observed the preliminary correlation between the changes (muscle hypertrophy) in contralateral extrinsics and intrinsics muscles at the same levels, due to biomechanical instability; local areas of spasticity (trigger points) were successfully detected on distal/proximal leg, pelvis and spine. Documented pictures were collected and accessible for telemedical consulting.

Conclusions: Extensive evaluation of motion posture in foot, ankle, and gluteus region, pelvis and spine is feasible and informative protocol for patient with pain and rheumatic diseases. Further studies needed to evaluate reliability studies, comparative RCT using US, CAREN, static and dynamic balance tests, pressure analysis, and extensive molecular profiling, to study crosslinks with immune phenotype of the patients; and to develop educational programs.

\section{REFERENCE:}

[1] Bubnov R Trigger Points Dry Needling Under Ultrasound Guidance for Low Back Pain Therapy. Comparative Study. Annals of the Rheumatic Diseases 2015;74:624. http://dx.doi.org/10.1136/annrheumdis-2015-eular.2323

Disclosure of Interest: None declared DOI: 10.1136/annrheumdis-2018-eular.3949

\section{AB1200 FROM THE CALCANEUS QUANTITATIVE ULTRASONOGRAPHY (QUS) TO THE FEMORAL RADIOFREQUENCY ECHOGRAPHIC MULTI SPECTROMETRY (REMS): NON-IONISING APPROACHES TO DIAGNOSIZE OSTEOPOROSIS PROPOSED BY F.I.R. M.O. FOUNDATION}

L. Cavalli ${ }^{\$ 1,2, *}$, L. Cianferotti ${ }^{\S 3}$, F. Giusti ${ }^{\$ 3}$, G. Gronchi ${ }^{3}$, P. Pisani ${ }^{2}$, M.L. Brandi ${ }^{3}$ ${ }^{1}$ Department of Surgery and Translational Medicine, University of Florence, Metabolic Bone Diseases Unit, University Hospital of Florence, Firenze; ${ }^{2}$ Institute of Clinical Physiology, National Research Council, Lecce; ${ }^{3}$ Department of Surgery and Translational Medicine, University of Florence, Metabolic Bone Diseases Unit, University Hospital of Florence, Florence, Italy, Firenze, Italy

Background: The high prevalence of osteoporosis and its insidious development, often silent until a fracture occurs, make it necessary to resort to prevention by promoting early diagnosis and educational programmes for a healthy life style. Objectives: To develop screening campaigns of the Italian population for the osteoporosis prevention thanks to the collaboration with F.I.R.M.O. Foundation (Fondazione Italiana Ricerca Malattie Ossee). ${ }^{1}$

Methods: An experienced medical staff administered to the afferent people the IOF "One minute risk test" questionnaire, (to detect the presence of clinical risk factors), together with a densitometric examination performed by a portable device aboard a mobile unit, in several Italian cities between 2011 and 2017.

The technique employed to assess bone status in 2011 and 2012 was calcaneus Quantitative Ultrasonography (QUS), applied to a peripheral skeletal site, which has been shown as effective in identifying osteoporotic men or post-menopausal women. ${ }^{2}$ Although representing a low-cost and accessible approach, the heel measurement of speed of ultrasound (SOS) can be influenced by foot positioning, oedema and temperature. ${ }^{3}$

Since 2017, a novel non-invasive densitometric technique is available, which allows to evaluate the axial fragile bone sites (spine and femur). It is Radiofrequency Echographic Multi Spectrometry (REMS), that a multicentric clinical study has been shown to provide parameters highly correlated with DXA ones.

Results: As measured by calcaneus QUS in 7305 subjects, the prevalence rate of osteoporosis was approximately $18.7 \%$, while the $42.6 \%$ had a T-score compatible with osteopenia. People with a QUS T-score $<-2.5$ was recommended to early undergo a DXA at lumbar and femoral sites and a specialistic visit. 